\title{
Text Classification using Graph Mining-based Feature Extraction
}

\author{
Chuntao Jiang, Frans Coenen, Robert Sanderson, and Michele Zito
}

\begin{abstract}
A graph-based approach to document classification is described in this paper. The graph representation offers the advantage that it allows for a much more expressive document encoding than the more standard bag of words/phrases approach, and consequently gives an improved classification accuracy. Document sets are represented as graph sets to which a weighted graph mining algorithm is applied to extract frequent subgraphs, which are then further processed to produce feature vectors (one per document) for classification. Weighted subgraph mining is used to ensure classification effectiveness and computational efficiency; only the most significant subgraphs are extracted. The approach is validated and evaluated using several popular classification algorithms together with a real world textual data set. The results demonstrate that the approach can outperform existing text classification algorithms on some dataset. When the size of dataset increased, further processing on extracted frequent features is essential.
\end{abstract}

Chuntao Jiang

The University of Liverpool, Department of Computer Science, Ashton Building, Ashton Street, Liverpool, L69 3BX, United Kingdom e-mail: c. jiang@liv. ac.uk

Frans Coenen

The University of Liverpool, Department of Computer Science, Ashton Building, Ashton Street, Liverpool, L69 3BX, United Kingdom e-mail: coenen@liv.ac.uk

Robert Sanderson

The University of Liverpool, Department of Computer Science, Ashton Building, Ashton Street, Liverpool, L69 3BX, United Kingdom e-mail: a zaroth@ liv . ac • uk

Michele Zito

The University of Liverpool, Department of Computer Science, Ashton Building, Ashton Street, Liverpool, L69 3BX, United Kingdom e-mail: mi chele@ liv . ac . uk 


\section{Introduction}

The most common document formalisation for text classification is the vector space model founded on the bag of words/phrases representation. The main advantage of the vector space model is that it can readily be employed by classification algorithms. However, the bag of words/phrases representation is suited to capturing only word/phrase frequency; structural and semantic information is ignored. It has been established that structural information plays an important role in classification accuracy [14].

An alternative to the bag of words/phrases representation is a graph based representation, which intuitively possesses much more expressive power. However, this representation introduces an additional level of complexity in that the calculation of the similarity between two graphs is significantly more computationally expensive than between two vectors (see for example [16]). Some work (see for example [12]) has been done on hybrid representations to capture both structural elements (using the graph model) and significant features using the vector model. However the computational resources required to process this hybrid model are still extensive.

The computational complexity of the graph representation for text mining is the main disadvantage of the approach, which prevents the full exploitation of the expressive power that the graph representation possesses. The work described in this paper seeks to address this issue by applying weighted graph mining analysis to the problem. The intuition behind the approach is that in standard frequent subgraph mining all generated subgraphs are assumed to have equal importance. However it is clear that, at least in the context of text mining, some subgraphs are more significant than others.

The rest of this paper is organized as follows. In Section 2 a brief overview of previous work is presented. The graph representation of document sets is then discussed in Section 3. In Section 4 the weighted subgraph mining is defined. The proposed Weighted graph mining algorithm, a variation of gSpan called Weighted gSpan (W-gSpan), is introduced in Section 5. A set of evaluating experiments are then presented in Section 6, followed by some concluding remarks in Section 7.

\section{Related Work}

Much early work on document graph representations for text classification was directed at Web documents. Geibel et al. in [7] demonstrated that it is possible to classify Web documents using document structure alone; however we shall demonstrate that a much more powerful approach is to combine structure with linguistic and semantic information. For example Schenker [16] proposed a number of methods to represent Web documents as graphs so as to include the structural information of the Web documents. The typical approach is to conduct classification using some similarity-based algorithm. However, approaches that operate using graph similarity measures are computationally expensive (for example computing the "maximum 
common subgraph" between two graphs is a NP hard problem [5]). Hybrid representations have been introduced to resolve the computational overhead associated with pure graph representations, see for example [12]. Such hybrid representations are reported to have better performance than pure graph based methods. However the computational resources required to process these hybrid model are still very high due to: (i) the extremely high number of nodes and edges, low number of edge labels and high repetition of structural node labels, encountered; and (ii) the consequent exponential complexity of the search space.

The use of graphs for representing text has a very long history in Natural Language Processing (NLP). However the work in NLP has focused on language understanding techniques such as Part Of Speech (POS) tagging, rather than text classification. Previous work $[13,20]$ has looked at the collocation of terms and their frequencies as graphs, rather than the linguistic structure of the sentence. One other study [6] has represented linguistic information as well as word order in a graph for text classification, however the work was limited to very small texts of between 8 to 13 tokens such as the titles of works. As such, we adopt the usage of of linguistic information, structure and semantics in a graph for text classification at a full text scale. In order to achieve this scale of processing, the use of frequent subgraph mining is essential.

Frequent subgraph (and sub-tree) mining, using various approaches, has been extensively studied $[9,10,22,8,2]$. However, the main bottleneck is the number of unnecessary candidate frequent subgraphs generated. A substantial amount of work has been undertaken focusing on developing efficient graph mining algorithms using elegant search strategies, data structures or their combinations. Some authors have suggested the use of constraint based frequent subgraph mining to remove unwanted patterns. The weighted subgraph mining approach advocated in this paper integrates the weight constraints into the frequent subgraph mining process to reduce the search space by generating only the most significant (interesting) patterns.

The frequent subgraph mining approach described in this paper is also influenced by work on weighted pattern mining, especially Weighted Association Rules Mining (WARM), see for example the work of [19, 17, 23, 24, 25]. A significant issue in WARM is that the "Downward Closure" (DC) property of items sets, on which many ARM algorithms are based, no longer holds. One solution (for example [19]) is to handle the weights as a post-processing step after mining frequent itemsets, however the weights are then not integrated into the ARM process. Tao et al. [17] proposed a model of weighted support, which satisfies a weighted DC property. Yun et al. [23, 24,25 ] introduced a series of concepts such as "weight range", "weight confidence", and "support confidence" for WARM in order to maintain the DC property and push the weight constraint deeply into the mining process. Although the ideas espoused by WARM cannot be directly applied to weighted frequent subgraph mining; the research described here is, at least in part, influenced by this body of work. 


\section{Graph Representation of Text Data}

The graph representation advocated in this paper is described in this section. The representation serves to capture a range documents aspects: (i) word stem, (ii) word Part Of Speech (POS), (iii) word order, (iv) word hypernyms, (v) sentence structure, (vi) sentence division and (vii) sentence order. There are four different types of nodes in the graph representation:

1. Structural: Nodes that represent sentences (S) and their internal structures of noun (NP), verb (VP) and prepositional phrases (PP). (Represented by triangles in Figure 1.)

2. Part of Speech: Nodes that represent the POS of a word, (eg. DT, JJ, NN). (Circles.)

3. Token: Nodes that represent the actual word tokens in the text. (Rectangles.)

4. Semantic: Nodes that represent additional information about the word such as its linguistic stem and other broader concepts. (Ovals)

Note that each node has a unique identifier and a label. There are also five types of edge in the graph:

1. hasChild: Edges which record the structure of the text such as a sentence having a noun phrase and a verb phrase or a noun phrase containing an adjective. (Unlabeled in Figure 1 for reasons of space.)

2. isToken: Edges which link the part of speech of a token to the token itself.

3. next: Edges which record the order of the words and sentences in the text.

4. stem: Edges which link to the linguistic stem of the word.

5. hyp: Edges which link to a broader concept.

An example of these node and edge types is depicted in Figure 1, using the first 6 words in a well known English sentence. Employing the above graph representation each sentence in each text is encoded and linked together with "next" edges to form one graph per text. Content based weightings were then attached to each node in the graph. The Structural elements, being intuitively unimportant to classification, were given a static low weight of 1 . The Part of Speech nodes were given a static weight of 10 , Token nodes were weighted according to their frequency in the dataset using the $T F \cdot I D F$ method. Stems were half the value of the Token and Hypernyms one quarter the value.

\section{Weighted Frequent Subgraphs}

In this section the weighted subgraph mining problem is formally defined. As with standard transaction graph mining approaches $[9,10,1,11]$ we commence with a set of transaction graphs $D=\left\{G_{1}, G_{2}, \cdots, G_{n}\right\}$ and a function $\tau(g, G)$ for arbitrary graphs $g$ and $G$. $\tau(g, G)=1$ (resp. 0), if $g$ is isomorphic to a subgraph in $G$. 


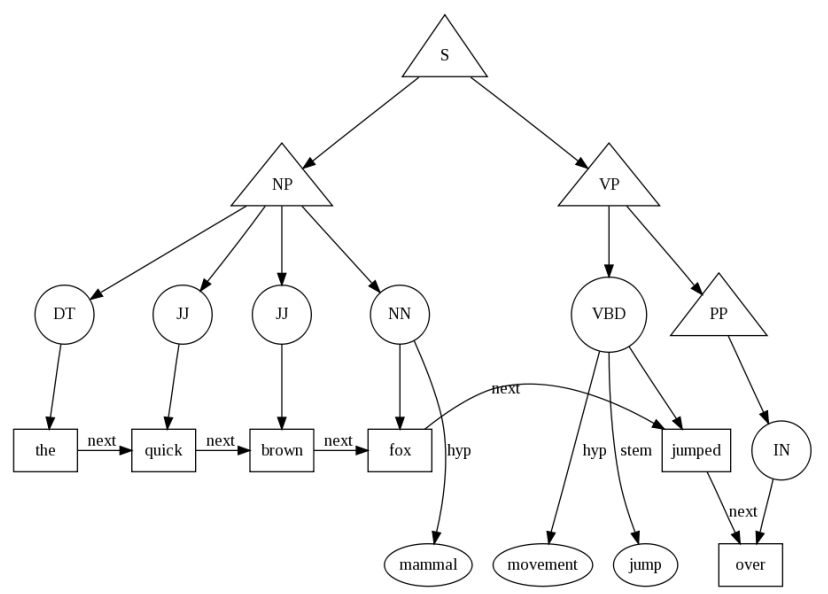

Fig. 1 Graph-based Text Representation Example

Definition 1. The support count of a graph (pattern) $g$ with respect to a database $D=\left\{G_{1}, G_{2}, \cdots, G_{n}\right\}$, is the expression $\operatorname{sco}(g)=\sum_{i=1}^{i=n} \tau\left(g, G_{i}\right)$. The support of $g$ with respect to $D, \sup (g)$, is the ratio of the support count over the size of the dataset $D$. Then:

$$
\sup (g)=\frac{\operatorname{sco}(g)}{n} .
$$

It should be remarked that $\operatorname{sco}(g)$ and $\sup (g)$, like most terms defined in this section depend on the dataset $D$. To avoid cluttering notations, such dependence will always be left implicit.

Definition 2. Given a graph $g$, if $\sup (g)$ is greater than or equal to some user defined minimum threshold $\theta$, then $g$ is said to be frequent (in $D$ ). The frequent subgraph mining problem is to find all the frequent subgraphs in the database $D$.

Since the purpose of this paper is to study weighted graph mining in the remainder of this section we define this concept precisely. From now on we assume that graphs come with weights associated with either their vertices or their edges. Let $W$ be a function assigning a weight to any graph $g$ in terms of the given weights for its vertices (resp. edges). In our work, in particular, $W$ will always be a sum of the vertex (resp. edge) weights, but the definitions in this Section hold in a more general setting.

Definition 3. Given a graph $g$ with the weight $W(g)$, the weighted support of $g$ with respect to $D, w \sup (g)$, is:

$$
\operatorname{wsup}(g)=W(g) \times \sup (g) .
$$

Definition 4. A graph $g$ is said to be weighted frequent if and only if its weighted support is greater than or equal to a given minimum support threshold (minwsup), 


$$
\operatorname{wsup}(g) \geq \operatorname{minwsup} .
$$

From (1), (2) and (3), a graph $g$ is weighted frequent if its support count satisfies:

$$
\operatorname{sco}(g) \geq \frac{\operatorname{minwsup} \times n}{W(g)}
$$

Note that $\operatorname{sco}(g)$ is always an integer. Hence we may define

$$
\operatorname{sbound}(g)=\left\lceil\frac{\text { minwsup } \times n}{W(g)}\right\rceil
$$

and we have

$$
\operatorname{sco}(g) \geq \operatorname{sbound}(g) .
$$

\section{Weighted gSpan}

The operation of the proposed weighted subgraph mining algorithm (W-gSpan) is described in this section. The section commences (Sub-section 5.1) with a discussion of support-bound candidate subgraph pruning. This is followed in Sub-section 5.2 by a description of a number of different weighting mechanisms that are used in this study. Sub-section 5.3 then gives the pseudo code of pruning algorithm and briefly decribes how W-gSpan is integrated into the classification process;

\subsection{Support Bound based Pruning}

Use of the DC property in any frequent set mining algorithm can greatly reduce the search space. However, in the context of weighted frequent set mining the DC property no longer holds. The W-gSpan algorithm therefore makes used of an alternative concept to prune non-interesting candidate subgraphs early on in the generation process.

Let the maximum possible size of a subgraph be $m L$ and the weight for a subgraph be defined as the sum of vertex weights (similar definitions may be given if the graph is edge-weighted). Given a $k$-pattern $g_{k}$ with weights $\left\{\omega_{1}, \omega_{2}, \cdots, \omega_{k}\right\}$, any future $n$-pattern containing $g_{k}$ is denoted by $g_{n}$, where $k<n \leq m L$. For the additional $(n-k)$ verticies, if the upper bounds of the weights are estimated as $\omega_{a_{k+1}}, \omega_{a_{k+2}}, \cdots, \omega_{a_{n}}$, then the upper bound of the weight of the $n$-pattern $g_{n}$ is given by:

$$
\text { wbound }_{n}\left(g_{k}\right)=\sum_{i=1}^{k} \omega_{i}+\sum_{i=k+1}^{n} \omega_{a_{i}}
$$


We may then define a lower bound of the support count of a $k$-pattern included in $g_{n}$ as

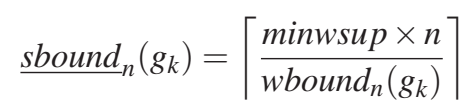

of course the definition can be extended to $n=k$ by setting $\underline{\operatorname{sbound}}_{k}\left(g_{k}\right)=\operatorname{sbound}\left(g_{k}\right)$ as defined in (5).

Definition 5. A $k$-subgraph $g_{k}$ is workable if $\operatorname{sco}\left(g_{k}\right) \geq$ sbound $_{n}\left(g_{k}\right)$ for some $n$ with $k \leq n \leq m L$, and unworkable if $\operatorname{sco}\left(g_{k}\right)<$ sbound $_{n}\left(g_{k}\right)$ for all $n$, with $k \leq n \leq m L$.

Lemma 1. If a subgraph $g_{k}$ is workable then it is possible for $g_{k}$ to be a subgraph of some weighted frequent n-subgraph. On the contrary, if a subgraph $g_{k}$ is not workable, then $g_{k}$ has no possibility of being a subgraph of any weighted frequent n-subgraph.

Proof. Let $n$ be given with $k \leq n \leq m L$. If $\operatorname{sco}\left(g_{k}\right) \geq$ sbound $_{n}\left(g_{k}\right)$, then due to $\operatorname{sco}\left(g_{k}\right) \geq \operatorname{sco}\left(g_{n}\right)$, it is possible that $\operatorname{sco}\left(g_{n}\right) \geq \operatorname{sbound}\left(g_{n}\right)$. So pattern $g_{n}$ has a chance to be weighted frequent in the future.

On the other hand, if $\operatorname{sco}\left(g_{k}\right)<\underline{s b o u n d}_{n}\left(g_{k}\right)$, then due to $\operatorname{sco}\left(g_{k}\right) \geq \operatorname{sco}\left(g_{n}\right)$, $\operatorname{sco}\left(g_{n}\right)<\operatorname{sbound}\left(g_{n}\right)$. So pattern $g_{n}$ will not be weighted frequent in the future.

The Weighted gSpan algorithm will then use a simple condition to decide whether or not to prune a particular $k$-pattern (in what follows $m L$ is the maximum length of a pattern):

if $\operatorname{sco}\left(g_{k}\right) \geq \operatorname{sbound}\left(g_{k}\right), g_{k}$ is workable; otherwise we compute $\underline{s b o u n d}_{m L}\left(g_{k}\right)$ (this gives

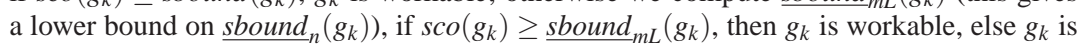
unworkable and pruned.

\subsection{Weight Calculation}

Given the notion of a weighted bound of a subgraph, as defined above, methods for calculating the weighting for a given subgraph are required. We can identify three approaches for determining subgraph weightings: (i) structure based, (ii) content based and (iii) structure and content based. The distinction between the two is that the structure base weighting approach does not require any advanced knowledge of the potential significance of subgraphs. Each approach is discussed in more detail below.

\subsubsection{Structure Based Weight Calculation}

In the structure base weighting approach weightings are derived purely from the "structure" of subgraphs. The approach advocated here is based on the frequency 
counts of individual nodes and edges per graph in the graph set. Using these frequency counts we adopt Pearson's Correlation Coefficient [15], PCC, to measure the weight of the edge (considering the nodes making up a 1-edge subgraph as two variables).Thus for two nodes $A$ and $B$, let the number of occurrences of $A$ equal $\phi_{A}$, the number of occurrences of $B$ equal $\phi_{B}$ and the number of co-occurrences of $A$ and $B$ equal $\phi_{A B}$; and let the total number of transaction graphs within the dataset be equal to $n$. The support values will then be $\sup (A)=\phi_{A} / n, \sup (B)=\phi_{B} / n$, and $\sup (A, B)=\phi_{A B} / n$. Using PCC the edge weight $\left(\omega_{p c c}\right)$ can be derived as follows.

$$
\omega_{p c c}=\frac{\sup (A, B)-\sup (A) \sup (B)}{\sqrt{\sup (A) \sup (B)(1-\sup (A))(1-\sup (B))}}
$$

Many other measures of association exist, such as the Chi Squared, cosine or Jaccard measure, that could equally well be used to determine edge weighting in a structured based context.

\subsubsection{Content based Weight Calculation}

In the content based weighting approach advanced knowledge of the nature of the input set is utilised. The nature of the advanced knowledge can take two forms: (i) weights that have been predefined (by for example a domain expert), or (ii) class labels associated with individual graph records (documents).

In the first case user supplied weightings can be attached directly to either nodes or edges. Thus given a set of user defined node weights $\omega_{1} \cdots \omega_{n}$, the weighting for a subgraph can be calculated by $\sum_{n_{i} \in g} \omega_{i}$. A similar calculation can be used in the event of user supplied edge weights. We later refer to this mechanism as the "Node Weight" method.

Alternatively we can calculate edge weights, given user defined node weights, as follows: if the nodes connecting edge $e_{i}$ are $a$ with weight $w_{a}$ and $b$ with $w_{b}$; the probability of $a$ 's occurrences is $\rho_{a}$, the probability of $b$ 's occurrences is $\rho_{b}$ and the probability of edge $e_{i}$ 's occurrences is $\rho(a, b)$. The mutual information metric between $a$ and $b$ can then be defined as $m u(a, b)=\rho(a, b) \log _{2}\left(\rho(a, b) / \rho_{a} / \rho_{b}\right)$. The weight for edge $e_{i}$ can then be calculated as:

$$
\omega_{e_{i}}=\left(\frac{2 \times w_{a} \times w_{b}}{w_{a}+w_{b}}\right) \times m u(a, b)
$$

The weight for the subgraph is calculated in the same manner as before. We refer to this mechanism as the "Mu" method.

Alternatively knowledge of the class label can be used to determine the weighting of a given subgraph. There are a number of feature selection techniques that can be utilised for this purpose, examples include Information Gain (IG), mutual information(MI), and $\chi^{2}$ testing. For the work described here the $\chi^{2}$ statistic was adopted to apply weightings to subgraphs according to their association with a given class label. Using the two-way contingency table of an edge $e$ and a graph's class label 
$y_{c}$, let $a$ denote the number of times $e$ and $y_{c}$ co-occur, $b$ denote the number of times the $e$ occurs without $y_{c}, c$ denote the number of times $y_{c}$ occurs without $e, d$ denote the number of times neither $e$ nor $y_{c}$ occurs, and $n$ is the total number of transaction graphs. The edge-goodness measure is then defined to be:

$$
\chi^{2}\left(e, y_{c}\right)=\frac{n(a d-c b)}{(a+c)(b+d)(a+b)(c+d)}
$$

The $\chi^{2}$ statistic has a value of zero if edge $e$ and class $y_{c}$ are indepedent. For each class $y_{c}$, we compute the $\chi^{2}$ statistic between each edge and that category, and then calculated the average value of $\chi^{2}$ statistic for each edge. Let $c=\left\{c_{1}, c_{2}, \cdots, c_{m}\right\}$ denote the set of categories for the transaction graphs dataset, $P_{r}\left(y_{c}\right)$ denotes the probability of $y_{c}$, then:

$$
\chi_{\text {avg }}^{2}(e)=\sum_{c=1}^{m} P_{r}\left(y_{c}\right) \chi^{2}\left(e, y_{c}\right)
$$

After estimating edge weights for each generated subgraph, the actual significance of the subgraph is calculated in the same manner as before. We refer to this mechanism as the "Chi Squared" method.

\subsubsection{Combined Content and Structure Based Weight Calculation}

It is possible to combine the two approaches, content and structure based weight calculation. For example given a user defined weight for node $n_{i}$ of $w_{n_{i}}$, then the probability of $n_{i}$ 's occurrences is $\rho$, and the entropy for node $n_{i}$ is entropy $\left(n_{i}\right)=$ $-\rho \log (\rho)-(1-\rho) \log (1-\rho)$. If we also make use of the "degree" (the number of edges incident to the node) of $n_{i}$ the weight for $n_{i}$ can be calculated as:

$$
\omega_{n_{i}}=w_{n_{i}} \times \operatorname{entropy}\left(n_{i}\right) \times \text { degree }\left(n_{i}\right)
$$

Thus, we refer to this mechanism as the "Entropy" method.

\subsection{The Weighted gSpan Algorithm (W-gSpan)}

The above weighting considerations were built into a variation of the well known gSpan frequent subgraph mining algorithm [22], Weighted gSpan (W-gSpan). However, the proposed weighing framework can equally well be applied to other frequent subgraph (or sub-tree) mining algorithms. The pseudo code for the pruning algorithm employed in W-gSpan is given in Algorithm 1.

After the W-gSpan algorithm is applied to identify weighted frequent subgraphs, these subgraphs are then used to generate a set of binary feature vectors(one per document). A standard classifier generator can then be employed using such vectors. 


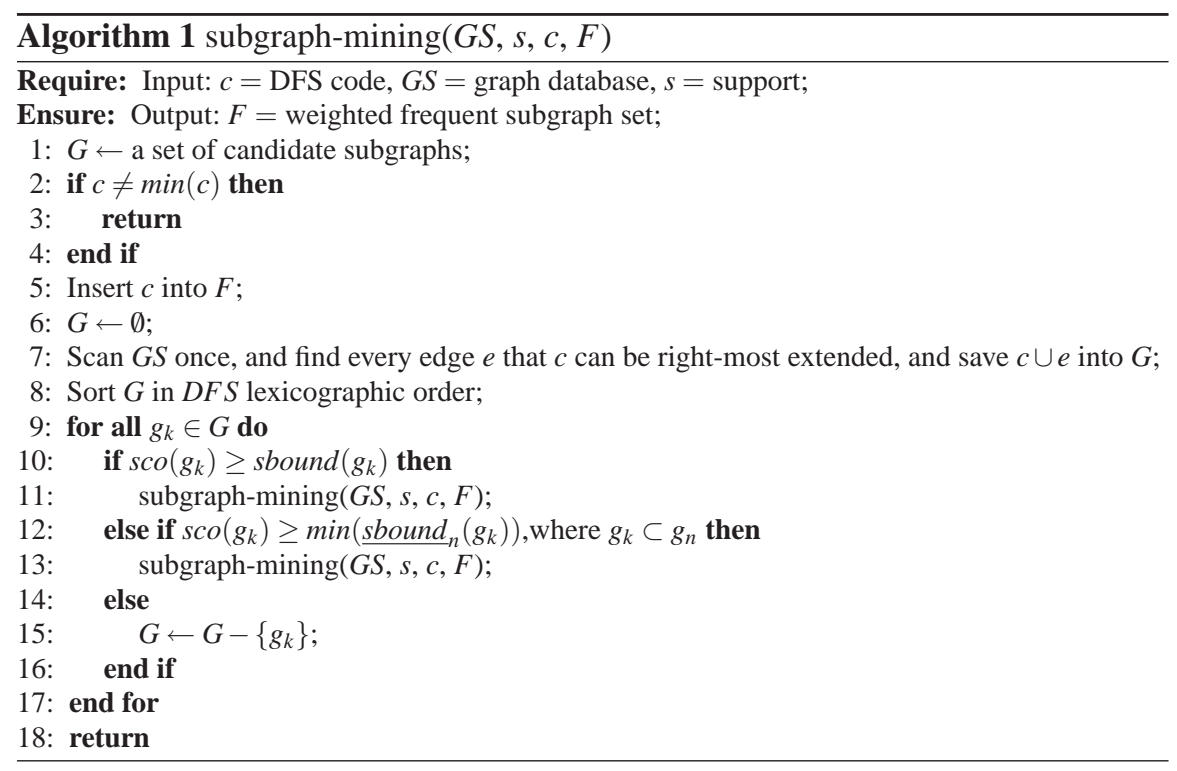

\section{Experiments and Results}

In order to evaluate the performance of the proposed graph based text classification method experiments were conducted to:

- Investigate the performance of W-gSpan, in terms of execution time and number of frequent subgraphs detected.

- Investigate the overall performance of the graph based classification process for text classification.

Note that the experiments were all run on a $1.86 \mathrm{GHZ}$ Intel Core $2 \mathrm{PC}$ with $2 \mathrm{~GB}$ main memory.

\subsection{Description of Text Data Set}

The experimental data consisted of three sets of documents(D1, D2, and D3) split evenly between two classes. The documents were extracted from the Medline dataset by their Medical Subject Heading (MeSH) fields, so that a two class ("polymerase chain reaction" and "magnetic resonance imaging") set was produced. The text was divided into sentences using a regular expression based tokenizer and then each sentence was POS tagged using Tsuruoaka and Tsujii's "geniatagger"[18], producing a sequence of "word/POS" tokens plus the lemma (stemmed form) of each word. This tagged output was then fed into a structural parser which produces a tree with noun, verb and prepositional phrases. The nouns and verbs are then "looked 
up" in the WordNet thesaurus and up to five broader terms added into the graph. The properties of the (graph) data are given in Table 1.

Table 1 Graph Data Description

\begin{tabular}{|l|lll|}
\hline \multicolumn{4}{|c|}{ Text Dataset } \\
\cline { 2 - 4 } & D1 & D2 & D3 \\
\hline \hline No. of graphs & 200 & 400 & 1000 \\
Maximal edge count & 3002 & 2917 & 4047 \\
Average edge count & 1141 & 1131 & 1135 \\
Distinct node label count & 10069 & 16456 & 26540 \\
\hline
\end{tabular}

\subsection{Performance of W-gSpan}

The performance of the W-gSpan algorithm was evaluateded using the four different weighting methods introduced in Sub-section 5.2 above:

- Pearson Correlation Coefficient ( $\mathrm{pcc}-\mathrm{w})$ for structure based weighting.

- Node Weight (node-w) for content based node weighting (Edge weighting would operate in a similar maner)

- Mutual information (mu-w) for content based edge weighting.

- Chi Square (chs-w) for content based class label discrimination weighting.

- Node entropy (entro-w) for combined structure and content based node weighting.

Experiments were also conducted with no weighting, but this was found to be extremely inefficient with poor outcomes, and thus are not discussed further in this evaluation.

The results of the performance experiments are presented in Figure 2. The runtime values corresponding to different minimum support thresholds are presented in Figure 2(a). The number of identified frequent subgraphs (features), corresponding to a range of minimum support thresholds, is presented Figure 2(b) and (c). There is, naturally, a correlation between support threshold and the number of identified subgraphs: as the support threshold is increased, the number of identified subgraphs decreases. There is also a natural correlation between runtime and the number of identified frequent subgraphs: runtime increases with the number of identified frequent subgraphs. From Figure 2(a) it can be observed that Mu weighting and node weighting seem to work well in terms of run time efficincy, however node weighting finds very few frequent subgraphs. The pcc weighting is the most effective in terms of computational efficiency, and works well in terms of number of features generated. Entropy weighting suddenly increases the runtime when the threshold is below $10 \%$. 

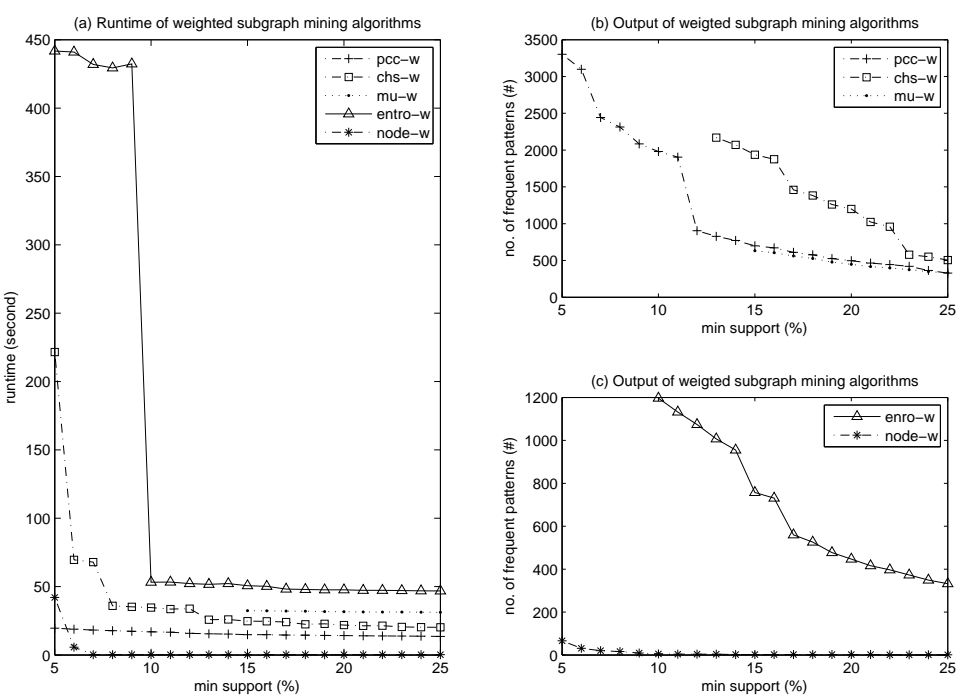

Fig. 2 Performance of weighted frequent subgraph mining on D1 dataset

\subsection{Classification Accuracy Comparison}

Three different classifier generator paradigms were use to evaluate the graph-based text classification process: (i) a classification association rule miner, TFPC [3, 4], (ii) a Naive Bayes Classifier (NBC) [21], and (iii) a decision tree classifier, C4.5 [21]. Table 2 shows the accuracy figures obtained using a range of support threshold values (for the generation of frequent subgraphs), for the three classification paradigms (with 10 folds cross validation) and using the five different weighting strategies. Experiments conducted with no weightings at all (on D1, D2 and D3 datasets) produced very poor results indicating, beyond doubt, that the proposed weighted graph mining approach provides genuine benefits.

Using no weighting on D1 dataset, it was not possible to obtain results with a support threshold below $85 \%$. When comparing the different weighting schemes, pcc produced the best overall accuracy. Using a standard 'bag of words' approach with TFPC gave a best accuracy of $89 \%$.

If the three classifier generators are compared, NBC performs significantly better than the other two, however C4.5 did not work well with any of the permutations of weighting and support threshold. If the three classifiers are applied on D2 and D3, the classification performance degrades. For example, using PCC weighting with support $10 \%$, the accuracy of NBC on D2 is $76.5 \%$ and the accuracy of NBC on D3 is $72.3 \%$. In order to get better accuracies, further processing on extracted frequent features is indispensable and how to model text data as more efficient graphs with less nodes and edges is also crucial. 
Text Classification using Graph Mining-based Feature Extraction

Table 2 Classification accuracy by different weighting methods on D1 dataset

\begin{tabular}{|l|l|lllllllllll|}
\hline \hline \multirow{2}{*}{ Classifier } & \multirow{2}{*}{ Method } & \multicolumn{8}{|c|}{ Support Threshold(\%) } \\
\cline { 2 - 10 } NBC & 15 & 16 & 17 & 18 & 19 & 20 & 21 & 22 & 23 & 24 & 25 \\
\hline \hline \multirow{5}{*}{ TFPC } & pcc-weight & 96.5 & 96 & 94.5 & 95 & 94 & 93 & 92 & 93 & 91.5 & 91.5 & 91.5 \\
& chs-weight & 91.5 & 91.5 & 89 & 87.5 & 86.5 & 86.5 & 90 & 91 & 90.5 & 92 & 91 \\
& mu-weight & 97 & 96.5 & 94.5 & 94 & 94 & 93 & 92 & 93 & 91.5 & 92 & 91.5 \\
& entro-weight & 76.5 & 75 & 95 & 95 & 94 & 92.5 & 92 & 92.5 & 92 & 93 & 92.5 \\
& node-weight & 80.5 & 80.5 & 80.5 & 80.5 & 80.5 & 80.5 & 80.5 & 80.5 & 80.5 & 80.5 & 80.5 \\
\hline \hline \multirow{5}{*}{ C4.5 } & pcc-weight & 91.5 & 91 & 88.5 & 89.5 & 86.5 & 84.5 & 83.5 & 84 & 84 & 84 & 84 \\
& chs-weight & 90.5 & 90 & 87.5 & 88.5 & 85.5 & 83.5 & 82.5 & 83 & 83.5 & 83 & 83 \\
& mu-weight & 92 & 91.5 & 89 & 90 & 87 & 85 & 84 & 84.5 & 84.5 & 84 & 84 \\
& entro-weight & 92 & 91.5 & 89 & 90 & 87 & 85 & 84 & 84.5 & 84.5 & 84 & 84 \\
& node-weight & 54 & 54 & 54 & 54 & 54 & 54 & 54 & 80.5 & 80.5 & 80.5 & 80.5 \\
\hline \hline \multirow{5}{*}{} & pcc-weight & 88.5 & 88 & 89.5 & 89 & 91 & 86.5 & 86.5 & 86.5 & 87 & 89 & 88.5 \\
& chs-weight & 87.5 & 87 & 89.5 & 89 & 91 & 86 & 86.5 & 86.5 & 87.5 & 89.5 & 89.5 \\
& mu-weight & 88.5 & 88 & 89.5 & 89 & 91 & 87 & 87 & 87 & 87 & 89 & 88.5 \\
& entro-weight & 88.5 & 88 & 89.5 & 89 & 91 & 87 & 87 & 87 & 87 & 89 & 88.5 \\
& node-weight & 80.5 & 80.5 & 80.5 & 80.5 & 80.5 & 80.5 & 80.5 & 80.5 & 80.5 & 80.5 & 80.5 \\
\hline
\end{tabular}

\section{Conclusion}

An approach to text classification using a graph based representation has been described. The graph representation of text allows both the structure and content of documents to be represented. Key constructs to support text classification can then be identified using frequent subgraph mining. The disadvantage of standard frequent subgraph mining is that it is computationally expensive, to the extent that any potential advantage of the graph representation of text cannot be realised. To overcome this disadvantage a weighted subgraph mining mechanism is proposed, W-gSpan. In effect W-gSpan selects the most significant constructs from the graph representation and uses these constructs as input for classification. Experimental evaluation demonstrates that the technique works well, significantly out-performing the unweighted approach in every case. A number of different weighting schemes were considered coupled with three different categories of classifier generator. In terms of the generated classification accuracy pcc-weighting outperformed the other proposed weighting mechanisms. PCC-weighting also worked well in terms of computational efficiency and therefore represents the best overall weighting strategies.

\section{References}

1. Cai, C. H., Fu, A. W., Cheng, C. H. and Kwong, W. W. Mining Association Rules with Weighted Items. In Proceedings of International Database Engineering and Applications Symposium, August 1998.

2. Chi, Y., Nijssen, S., Muntz, R. and Kok, J. Frequent Subgree Mining An Overview. In Fundamenta Informaticae, Special Issue on Graph and Tree Mining, 66(1-2), 161-198, 2005. 
3. Coenen, F. The LUCS-KDD TFPC Classification Association Rule Mining Algorithm. http://www.csC.Iiv.ac.uk/ frans/KDD/Software/Apriori_TFPC/ apriori TFPC. html, Dept. of Computer Science, The University of Liverpool, UK, 2004.

4. Coenen, F., Leng, P. Obtaining Best Parameter Values for Accurate Classification. In Proceedings of International Conference on Data Mining, Pages: 597-600, 2005.

5. Garey, M. R. and Johnson, D. S. Computers and Intractability - A Guide to the Theory of NP-Completeness. W. H. Freeman and Company, New York, 1979.

6. Gee, K. R. and Cook, D. J. Text Classification Using Graph-Encoded Linguistic Elements, In FLAIRS Conference 2005, pp. 487-492.

7. Geibel, P., Krumnack, U., Pustylnikow, O., Mehler, A., et al. Structure-Sensitive Learning of Text Types, In AI 2007: Advances in Artificial Intelligence, Vol 4830, pp. 642-646.

8. Huan, J., Wang, W. and Prins, J. Efficient Mining of Frequent Subgraph in the Presence of Isomorphism. In Proceedings of the 2003 International Conference on Data Mining, 2003.

9. Inokuci, A., Washio, T. and Motoda, H. An Apriori-based Algorithm for Mining Frequent Substructures from Graph Data. In Proceedings of the 4th European Conference on Principles and Practice of Knowledge Discovery in Databases, Pages: 13-23, 2000.

10. Kuramochi, M. and Karypis, G. Frequent Subgraph Discovery. In Proceedings of 2001 IEEE International Conference on Data Mining, 2001.

11. Lee, S. D. and Park, H. C. Mining Weighted Frequent Patterns from Path Traversals on Weighted Graph. In IJCSNS International Journal of Computer Science and Network Security, VOL.7, No.4, April 2007.

12. Markov, A., Last, M. Efficient Graph-based Representation of Web Documents. In Proceedings of the Third International Workshop on Mining Graphs, Trees and Sequences, Pages: 52-62, Porto Portugal, 2005.

13. Markov, A., Last, M. and Kandel, A. Fast Categorization of Web Documents represented by Graphs, In Advances in Web Mining and Web Usage Analysis, Vol 4811, pp. 56-71, 2007.

14. Mukund, D., Kuramochi, M. and Karypis, G. Frequent Sub-structure based Approaches for Classifying Chemical Compounds. In Proceedings of the Third IEEE International Conference on Data Mining, 2003.

15. Reynolds, H. T. The Analysis of Cross-classifications. The Free Press, New York, 1977.

16. Schenker, A. Graph Theorectic Techniques for Web Content Mining. PhD thesis, University of South Florida, 2003.

17. Tao, F., Murtagh, F. and Farid, M. Weighted Association Rule Mining using Weighted Support and Significance Framework. InProceedings of ACM SIGKDD International Conference on Knowledge Discovery and Data Mining, USA, Aug. 2003.

18. Tsuruoka, Y. and Tsujii, J. Bidirectional Inference with the Easiest-First Strategy for Tagging Sequence Data. In Proceedings of HLT/EMNLP 2005, pp. 467-474.

19. Wang, W., Yang, J. and Yu, P. S. Efficient Mining of Weighted Association Rules(WAR). In Proceedings of ACM SIGKDD International Conference on Knowledge Discovery and Data Mining, USA, Aug. 2000.

20. Wang, W., Do, D. B. and Lin, X. Term Graph Model for Text Classification, In Advanced Data Mining and Applications, pp. 19-30, 2005.

21. Witten, Ian H. and Frank, Eibe. Data Mining: Practical Machine Learning Tools and Techniques (2nd Edition). Morgan Kaufmann, San Francisco, 2005.

22. Yan, X. and Han, J. gSpan: Graph-based Substructure Pattern Mining. In Proceedings of 2002 International Conference on Data Mining, 2002.

23. Yun, U. and Leggett, J. J. WFIM: Weighted Frequent Itemset Mining with a Weight Range and a Minimum Weight. InProceedings of the Fifth SIAM International Conference on Data Mining, Pages: 636-640, April 2005.

24. Yun, U. and Leggett, J. J. WIP: Mining Weighted Interesting Patterns with a Strong Weight and/or Support Affinity. In Proceedings of the Sixth SIAM International Conference on Data Mining, 2006.

25. Yun, U. WIS: Weighted Interesting Sequential Pattern Mining with a Similar Level of Support and/or Weight. ETRI Journal, Vol. 29, No. 3, Pages: 336-352, June 2007. 